\title{
Diacronie
}

Studi di Storia Contemporanea

$\mathrm{N}^{\circ} 29,1 \mid 2017$

"Crash test"

\section{Matteo Pasetti, L'Europa corporativa. Una storia transnazionale tra le due guerre mondiali}

\section{Camilla Poesio}

\section{Q OpenEdition \\ 1 Journals}

Edizione digitale

URL: http://journals.openedition.org/diacronie/5095

DOI: $10.4000 /$ diacronie. 5095

ISSN: 2038-0925

Editore

Association culturelle Diacronie

Notizia bibliografica digitale

Camilla Poesio, « Matteo Pasetti, L'Europa corporativa. Una storia transnazionale tra le due guerre mondiali », Diacronie [Online], № 29, 1 | 2017, documento 17, Messo online il 29 mars 2017, consultato

il 23 septembre 2020. URL : http://journals.openedition.org/diacronie/5095; DOI : https://doi.org/

10.4000/diacronie.5095

Creative Commons License 


\section{Diacronie}

Studi di Storia Contemporanea

29, 1/2017

"Crash test". Continuità, discontinuità, legami e rotture nelle dinamiche della storia contemporanea

\section{RECENSIONE: Matteo PASETTI, L'Europa corporativa. Una storia transnazionale tra le due guerre mondiali, Bologna, Bononia University Press, 2016, 336 pp.}

A cura di Camilla POESIO

Per citare questo articolo:

POESIO, Camilla, «RECENSIONE: RECENSIONE: Matteo PASETTI, L'Europa corporativa. Una storia transnazionale tra le due guerre mondiali, Bologna, Bononia University Press, 2016, 336 pp.», Diacronie. Studi di Storia Contemporanea : "Crash test". Continuità, discontinuità, legami e rotture nelle dinamiche della storia contemporanea, 29, 1/2017, 29/3/2017,

URL: <http://www.studistorici.com/2017/3/29/poesio_numero_29/ >

Diacronie Studi di Storia Contemporanea $\rightarrow$ http://www.diacronie.it Rivista storica online. Uscita trimestrale.

redazione.diacronie@hotmail.it

Comitato di direzione: Naor Ben-Yehoyada - João Fábio Bertonha - Christopher Denis-Delacour - Maximiliano Fuentes Codera Anders Granås Kjøstvedt - John Paul Newman - Deborah Paci - Niccolò Pianciola - Spyridon Ploumidis - Wilko Graf Von Hardenberg

Comitato di redazione: Jacopo Bassi - Luca Bufarale - Gianluca Canè - Fausto Pietrancosta - Alessandro Salvador - Matteo Tomasoni Diritti: gli articoli di Diacronie. Studi di Storia Contemporanea sono pubblicati sotto licenza Creative Commons 3.0. Possono essere riprodotti e modificati a patto di indicare eventuali modifiche dei contenuti, di riconoscere la paternità dell'opera e di condividerla allo stesso modo. La citazione di estratti è comunque sempre autorizzata, nei limiti previsti dalla legge. 


\title{
17/ RECENSIONE: Matteo PASETTI, L'Europa corporativa. Una storia transnazionale tra le due guerre mondiali, Bologna, Bononia University Press, 2016, 336 pp.
}

\author{
A cura di Camilla POESIO
}

La prima impressione che si ha leggendo questo denso e complesso libro è quella di avere fra le mani un testo destinato a essere di riferimento tanto per gli studi sul tema del corporativismo, tanto per gli studi sul concetto di transnazionale in generale. Autore di numerose pubblicazioni sulla storia del Novecento, in particolare sulla storia del movimento sindacale ${ }^{1}$ e sul fascismo italiano anche in ottica europea ${ }^{2}$, Pasetti è da anni studioso del corporativismo nella sua versione fascista ${ }^{3}$. In questa sua ultima fatica, l'autore affronta il tema sia come oggetto di studio in sé e per sé sia, soprattutto, come esempio di storia transnazionale. Il libro che abbiamo davanti non si occupa, perciò, solo di corporativismo fascista - che comunque viene spiegato nello specifico in un paragrafo a parte ${ }^{4}$ - quanto della sua circolazione in Europa (ma non solo):

esso [il corporativismo fascista] sarà osservato in "movimento", seguendo cioè non solo il suo sviluppo, quanto soprattutto la ricezione all'estero e l'interazione con altri progetti ed esperienze $e^{5}$.

La scelta di affrontare il corporativismo da un punto di vista transnazionale, spiega l'autore, non è stata presa ritenendo che tale prospettiva sia più valida di altre, ma è stata dettata dalla natura stessa del corporativismo la cui storia, come emerge dal libro, fu essa stessa transnazionale. Infatti, nell'interessante introduzione, viene portata avanti una lunga

\footnotetext{
${ }^{1}$ PASETTI, Matteo, Tra classe e nazione. Rappresentazioni e organizzazione del movimento nazional-sindacalista (1918-1922), Roma, Carocci, 2008.

${ }^{2}$ ID., Storia dei fascismi in Europa, Bologna, Archetipolibri, 2009.

${ }^{3}$ ID. (a cura di), Progetti corporativi tra le due guerre mondiali, Roma, Carocci, 2006; ID., Neither Bluff nor Revolution. The Corporations and the Consolidation of the Fascist Regime, in ALBANESE, Giulia, PERGHER, Roberta (eds.), In the Society of Fascists: Acclamation, Acquiescence and Agency in Mussolini's Italy, New York, Palgrave Mac Millan, 2012, pp. 87-107.

${ }^{4}$ PASETTI, Matteo, L'Europa corporativa. Una storia transnazionale tra le due guerre mondiali, Bologna, Bonomia University Press, 2016, pp. 113-125.

${ }^{5}$ Ibidem, p. 19.
} 
disquisizione storicizzata del concetto polisemico di corporativismo ${ }^{6}$ e il solo fatto che molteplici siano stati gli usi storici del termine, che peraltro ha cambiato di significato in alcune lingue, ci porta a quanto detto sopra, e cioè, che la dottrina politica del corporativismo è un esempio di storia transnazionale: le idee corporativiste hanno viaggiato, si sono propagate, sono state accolte, tradotte, interpretate, riadattate e trasformate con altre esperienze e progetti finendo talvolta per diventare altro dall'originale.

C'è anche un terzo livello con cui questo libro, secondo noi, può essere letto: analizzando il corporativismo nella sua dimensione transnazionale e soffermandosi sulle influenze politiche e socio-economiche che ebbe il fascismo italiano su altri paesi, emerge ancora una volta e con chiarezza la necessità - e Pasetti lo fa - di leggere e inquadrare la storia del fascismo italiano in relazione alla situazione europea ${ }^{7}$.

Un'altra originalità del volume sta nel fatto che, se la maggior parte della storiografia ha analizzato l'interesse dell'opinione pubblica europea (e non solo) per il corporativismo negli anni Trenta come possibile terza via in seguito alla crisi del 1929 e alla politica propagandistica del regime fascista, Pasetti, senza tralasciare quel decennio, affronta anche l'interesse e la seduzione che quella dottrina provocò prima, già nella seconda metà degli anni Venti. L'interesse per la ricezione all'estero del corporativismo serve a Pasetti «non tanto per ricostruire un inesistente "spirito del tempo" [...] quanto per comprendere alcune dinamiche che portarono all'effettiva circolazione transnazionale di un modello» ${ }^{8}$.

In accordo con alcuni dei più recenti studi sul tema del corporativismo che hanno rivalutato l'importanza delle corporazioni nel funzionamento del regime ${ }^{9}$, Pasetti dimostra, facendo uso di una ricca mole di fonti a stampa e di fonti primarie straniere, ossia di testi coevi ai fatti, quanto il corporativismo fascista produsse degli effetti niente affatto trascurabili. Senza smentire le argomentazioni di un Gaetano Salvemini o di un Edward Carr, e cioè il sostanziale fallimento pratico del corporativismo ${ }^{10}$, emerge il fatto rilevante che il corporativismo fascista fu argomento di discussione di centinaia di intellettuali e politici in Europa e anche in America del Sud e negli

\footnotetext{
${ }^{6}$ Ibidem, pp. 11-18.

${ }^{7}$ In questa prospettiva vanno menzionati alcuni dei più recenti studi, tra cui RODRIGO, Javier (ed.), Políticas de la violencia. Europa, siglo XX, Zaragoza, Prensas de la Universidad de Zaragoza, 2014, pp. 81-115.

${ }^{8}$ PASETTI, Matteo, L'Europa corporativa, cit., p. 127.

${ }^{9}$ GAGLIARDI, Alessio, Il corporativismo fascista, Roma-Bari, Laterza, 2010; CASSESE, Sabino, Lo Stato fascista, Bologna, Il Mulino, 2010. Per una diversa interpretazione cfr. SANTOMASSIMO, Gianpasquale, La terza via fascista. Il mito del corporativismo, Roma, Carocci, 2006.

${ }^{10}$ Cfr. SALVEMINI, Gaetano, Sotto la scure del fascismo, in ID., Scritti sul fascismo, vol. III, Milano, Feltrinelli, 1974, [Ed. orig.: Under the Axe of Fascism, London, Victor Gollancz, 1936], p. 118; CARR, Edward, Sei lezioni sulla storia, Torino, Einaudi, 1966 (citato in PALLA Marco, Fascismo e Stato corporativo. Un'inchiesta della diplomazia britannica, Milano, Franco Angeli, 1991, p. 117).
} 
Stati Uniti, perciò, "che fosse o meno un "bluff", o una "truffa", in molti presero sul serio l'esperimento fascista, e questo fatto non può essere considerato del tutto irrilevante [...]» .

È partendo da questa convinzione, che il libro si snoda in tre corposi capitoli, a loro volta suddivisi in numerosi paragrafi che ci conducono addentro la questione.

Il primo capitolo affronta la nascita di progetti corporativi nella cultura politica del primo dopoguerra e le varie teorie sul rilancio del corporativismo per ridefinire i rapporti tra stato, economia e società, come quelle esposte tra il 1917 e il 1919 da Walter Rathenau. La prima guerra mondiale aveva travolto il vecchio ordine politico liberale e il tema del corporativismo ritornò in particolare in tre correnti politiche: l'area del cattolicesimo politico, l'area nazionalista e l'area del socialismo corporativo o guild socialism.

Il "revival", la diffusione di ipotesi variamente corporative che, con una circolazione transnazionale, attraversarono il dibattito politico europeo, condusse dunque un attacco su più fronti alla sovranità dello stato parlamentare, investendo l'ordine istituzionale, i meccanismi della rappresentanza politica dei gruppi sociali, i rapporti con le organizzazioni degli interessi, la regolamentazione degli scambi tra potere economico e potere politico ${ }^{12}$.

Ebbero luogo alcuni tentativi di messa in pratica di riforme di tipo corporativo che tuttavia fallirono. I due esperimenti più espliciti di rifondare la democrazia parlamentare su basi corporative furono in Portogallo e a Fiume. Nel paese iberico, Sidónio Pais, salito al potere con un colpo di stato nel 1917, prefigurò una riforma costituzionale che modificava l'assetto parlamentare con la creazione di una camera in parte corporativa: primo caso in Europa di un'assemblea istituzionale nazionale in cui i rappresentanti di categorie professionali potevano prendere iniziative legislative. L'altro tentativo costituzionale di stampo corporativo che fallì fu quello di Fiume espresso nella Carta del Carnaro che offriva un'alternativa all'ordinamento bolscevico e a quello democratico borghese prospettando una sorta di "democrazia corporativa". Seguirono poi altri esperimenti che, invece, non mirarono a sostituire il parlamentarismo bensì ad affiancargli "consigli economici" o "comitati paritari" per intervenire sui processi di politica economica e risolvere la conciliazione tra le parti sociali per mezzo di meccanismi istituzionalizzati: successe in Inghilterra, in Spagna, in Belgio, nella Repubblica di Weimar - prima con la Zentralarbeitsgemeinschaft poi con il Reichswirtschaftsrat - e in Francia.

Rispetto a questi primi esperimenti, il regime fascista «fornì una soluzione più radicale, coercitiva e illiberale a problemi di governance che erano comuni a tutte le società di massa occidentali e che vennero affrontati con modalità per certi aspetti analoghe, cioè, tramite la

\footnotetext{
${ }^{11}$ PASETTI, Matteo, L'Europa corporativa, cit. p. 26.

${ }^{12}$ Ibidem, p. 58.
} 
prassi definita dall'idealtipo del "corporativismo"13". Il corporativismo divenne una sorta di cavallo di Troia per alcuni esponenti del movimento fascista finendo per inserirsi in circuiti intellettuali e politici europei. Accadde così per Camillo Pellizzi, fondatore della sezione inglese dei fasci italiani all'estero, che tramite il tema del corporativismo entrò in contatto anche con ambienti del laburismo.

Il secondo capitolo affronta la svolta fascista: il 21 aprile 1927 fu emanata la Carta del Lavoro, elaborata da Alfredo Rocco, che fissò i principi della politica corporativa del regime, essenzialmente tre: abolizione del diritto di sciopero e di serrata e istituzione di una Magistratura del lavoro per la soluzione delle controversie; riconoscimento di un'unica associazione fascista per ogni categoria dei lavoratori; costituzione del ministero delle Corporazioni e del Consiglio nazionale delle corporazioni.

Perché, tra i vari elementi ideologici del fascismo, il corporativismo fascista fu il più condiviso? Perché era il più facilmente estrapolabile e perché sembrava risolvere con la creazione di nuove organizzazioni corporative la lotta di classe attraverso la Confederazione nazionale delle corporazioni sindacali. Molti ne rimasero affascinati, e non solo chi afferiva all'area di estrema destra, bensì anche certi ambienti del cattolicesimo intransigente e alcune minoranze socialiste $\mathrm{e}$ democratiche. Tra la fine degli anni Venti e l'inizio degli anni Trenta dell'esperimento corporativo in atto in Italia si parlò molto in numerose testate all'estero, in Spagna, Francia, Belgio, Svizzera, Austria, Germania, Ungheria, Croazia, Cecoslovacchia, Bulgaria, Romania, Ucraina, addirittura in Brasile; una notevole mole di libri fu tradotta nelle lingue più parlate. Anche all'interno della Organizzazione internazionale del lavoro alcuni espressero autentico interesse per la Carta del lavoro, come nel caso del Direttore generale dell'OIL stessa, Albert Thomas. Tutto ciò dimostra quello che Pasetti chiama " "il potenziale viaggiante" del fascismo italiano come esperienza capace di attraversare i confini porosi di varie aree ideologiche, allargando la propria sfera di influenza ben oltre movimenti affini o dichiaratamente fascisti ${ }^{14}$. E sebbene questi ambienti fossero sostanzialmente ambienti accademici, e dunque questa circolazione avesse un carattere sostanzialmente elitario, ciò non significa, per Pasetti, che l'opinione corporativa ebbe un ruolo irrilevante nella cultura politica e nelle riforme istituzionali dell'epoca.

Un primo esempio di applicazione e contemporaneamente di riadattamento, dunque, di ibridazione dell'esperimento corporativo italiano fu quello effettuato durante la dittatura di Miguel Primo de Rivera quando la monarchia spagnola si dotò con l'istituzione dell'Organización nacional corporativa, il cui progetto fu affidato a Eduardo Aunós Pérez, di un'organizzazione corporativa, per molti aspetti simile a quella italiana. Il caso spagnolo è un chiaro esempio di come il corporativismo fu transnazionale: la riforma di Perez, infatti, aveva tratto ispirazione non

\footnotetext{
${ }^{13}$ Ibidem, p. 90.

${ }^{14}$ Ibidem, p. 153.
} 
solo dal modello italiano, ma, per ammissione dello stesso Perez, anche dai comitati paritetici istituiti in Belgio.

L'esperienza del corporativismo funzionò per il regime fascista come fonte di legittimazione del regime stesso e sul corporativismo fu portata avanti una notevole promozione all'estero, una sorta di «operazione di marketing», tanto che dal 1927 il corporativismo divenne un Leitmotiv dell'attività di propaganda all'estero effettuata tramite vari soggetti (i Fasci all'estero, i Caur, la Società Dante Alighieri, i vari istituti di cultura). La Grande crisi economica fece il resto, funzionando da cassa di risonanza in favore del corporativismo.

Il terzo e ultimo capitolo esamina quello che Pasetti chiama gli "avatars", "sia nel senso di replicanti, cloni dell'archetipo fascista, sia in quello di autorappresentazioni utilizzate per dissimulare la natura di regimi impegnati in processi, per certi aspetti contraddittori, di fascistizzazione» ${ }^{15}$. Si tratta, cioè, di esperienze che indubbiamente presero a modello il caso italiano, ma da cui si allontanarono inserendo caratteristiche autoctone, come il caso esemplificativo dell'Argentina studiato da Federico Finchelstein ${ }^{16}$; ma è soprattutto al Portogallo di Salazar - il primo stato nell'Europa degli anni Trenta a darsi una costituzione corporativa -, all'Austria di Dolfuss, alla Polonia di Piłsudski, alla Spagna di Franco, alla Grecia di Metaxas, alle dittature della Bulgaria e della Romania, alla Slovacchia di monsignor Tiso e al regime di Vichy che Pasetti pensa quando parla di "avatars". In tutti questi casi, differenti gli uni dagli altri, a partire dal 1933, indubitabile fu l'influenza dell'esperienza corporativa italiana, ma ogni regime adattò il modello alle proprie specificità ed esperienze autoctone, dando vita, di fatto, a un "prodotto" diverso dall'originale: «poiché la legislazione fascista non era più l'unico prototipo esistente, ogni "avatar" divenne un ulteriore possibile riferimento per gli altri» ${ }^{17}$. Un esempio di ciò fu l'esperimento portoghese salazarista che divenne un modello alternativo a quello italiano.

Un caso del tutto a parte fu la Germania nazista: la Legge sull'ordinamento del lavoro nazionale del 1934 segnò la fine di qualsiasi velleità corporativa e fornì un modello diverso di disciplina dei rapporti sociali, istituendo una regolazione contrattuale tramite l'ordinamento aziendale (Betriebsordnung) su cui di fatto decideva il capo dell'azienda, previa autorizzazione dei fiduciari nazionali del lavoro di nomina ministeriale, mentre al Fronte del lavoro tedesco (Deutsche Arbeitsfront), l'organizzazione unitaria istituita dopo lo scioglimento dei sindacati, furono affidate funzioni solo consultive.

Leggendo questo libro, complesso nei contenuti ma facilitato da una scrittura asciutta e mai ridondante, si rimane meravigliati dalla ricca bibliografia utilizzata, dalla mole di documenti

\footnotetext{
${ }^{15}$ Ibidem, p. 28.

${ }^{16}$ FINCHELSTEIN Federico, Transatlantic Fascism. Ideology, Violence, and the Sacred in Argentina and Italy, 19191945, Durham-London, Duke University Press, 2010.

${ }^{17}$ PASETTI, Matteo, L'Europa corporativa, cit., p. 258.
} 
visionati e dallo sforzo di sintesi che è stato senza dubbio fatto: siamo certi che il volume si collocherà tra i testi di riferimento per la tematica del corporativismo, a cui apporta nuova linfa valorizzando la dimensione europea del fenomeno. 


\section{L'AUTORE}

Camilla POESIO si è addottorata presso l'Università Ca' Foscari di Venezia in cotutela con la Freie Universität Berlin; collabora con l'Università di Paderborn e da gennaio 2017 sarà Visiting Fellow presso il Department of History and Civilization del European University Institute; è Cultore della materia presso il DSLCC-Università Ca' Foscari Venezia. Ha vinto con la sua tesi di dottorato il Premio Nicola Gallerano 2009 e il Premio Ettore Gallo 2009. È autrice dei volumi Reprimere le idee, abusare del potere. La Milizia e l'instaurazione del regime fascista (Roma Aracne, 2010); Il confino fascista. L'arma silenziosa del regime, (Roma-Bari, Laterza, 2011) e ha pubblicato saggi su numerose riviste. I suoi interessi sono focalizzati sulla storia dell'Italia e della Germania del XX secolo in prospettiva comparata e transnazionale, $\mathrm{i}$ rapporti fra fascismo italiano e nazismo, i diritti umani, l'uso della violenza, la memoria pubblica e privata, il jazz e la musica nel fascismo.

URL: < http://www.studistorici.com/progett/autori/\#Poesio > 